\title{
BMJ Open Incidence of renal replacement therapy for diabetic nephropathy in the Netherlands: Dutch diabetes estimates (DUDE)-3
}

Peter R van Dijk, ${ }^{1}$ Anneke Kramer, ${ }^{2}$ Susan J J Logtenberg, ${ }^{1,3}$ Andries J Hoitsma, ${ }^{4,5}$ Nanne Kleefstra, ${ }^{1,3}$ Kitty J Jager, ${ }^{2}$ Henk J G Bilo ${ }^{1,3,6}$

To cite: van Dijk PR, Kramer A, Logtenberg SJJ, et al. Incidence of renal replacement therapy for diabetic nephropathy in the Netherlands: Dutch diabetes estimates (DUDE)-3. BMJ Open 2015;5:e005624. doi:10.1136/bmjopen-2014005624

- Prepublication history and additional material is available. To view please visit the journal (http://dx.doi.org/ 10.1136/bmjopen-2014005624).

Received 5 May 2014 Revised 25 August 2014 Accepted 26 August 2014

CrossMark

For numbered affiliations see end of article.

Correspondence to

Peter R van Dijk;

P.r.van.Dijk@isala.nl

\section{ABSTRACT}

Objectives: Describe the incidence, prevalence and survival of patients needing renal replacement therapy (RRT) for end-stage renal disease (ESRD) due to diabetes mellitus (DM)-related glomerulosclerosis or nephropathy (diabetic nephropathy, DN) in the Netherlands.

Design: Using the national registry for RRT (RENINEregistry), data of all Dutch individuals initiating RRT for ESRD and having DN as primary diagnosis in the period 2000-2012 were obtained.

Setting: Observational study in the Netherlands. Patients: Patients with ESRD needing RRT for DN. Outcome measurements: Age and gender adjusted incidence and prevalence of RRT for DN in the period 2000-2012. In addition, trends in time and patient's survival were examined.

Results: The prevalence of DM in the general population increased from approximately 466000 in 2000 to 815000 in 2011. The number of individuals who started RRT with DN as primary diagnosis was 17.4 per million population ( $\mathrm{pmp}$ ) in 2000 and $19.1 \mathrm{pmp}$ in 2012, with an annual percentage change (APC) of $0.8 \%(95 \% \mathrm{Cl}-0.4$ to 2.0$)$. For RRT due to type $1 \mathrm{DN}$, the incidence decreased from 7.3 to $3.5 \mathrm{pmp}$ (APC $-4.8 \%, 95 \% \mathrm{Cl}-6.5$ to -3.1 ) while it increased for type $2 \mathrm{DN}$ from 10.1 to $15.6 \mathrm{pmp}$ (APC $3.1 \%, 95 \% \mathrm{Cl} 1.3$ to 4.8 ). After 2009 , the prevalence of RRT for DN remained stable (APC $1.0 \%, 95 \% \mathrm{Cl}$ -0.4 to 2.5). Compared to the period 2000-2004, patients initiating RRT and dialysis in 2005-2009 had better survival, HRs $0.8(95 \% \mathrm{Cl} 0.7$ to 0.8$)$ and 0.8 $(95 \% \mathrm{Cl} 0.7$ to 0.9$)$, respectively, while survival after kidney transplantation remained stable, $\mathrm{HR} 0.8,95 \% \mathrm{Cl}$ 0.5 to 1.1 .

Conclusions: Over the last decade, the incidence of RRT for DN was stable, with a decrease in RRT due to type $1 \mathrm{DN}$ and an increase due to type $2 \mathrm{DN}$, while survival increased.

\section{INTRODUCTION}

Diabetic nephropathy (DN) is considered to be the main cause of end-stage renal disease

\section{Strengths and limitations of this study}

- This study reports the incidence, prevalence and survival of patients needing renal replacement therapy (RRT) for end-stage renal disease (ESRD) due to diabetic nephropathy (DN).

- Nationwide data from 2000 to 2012 for DN due to both type 1 and type 2 diabetes mellitus are reported.

- It cannot be ruled out that some of the patients with unknown or missing primary causes of RRT actually had RRT for ESRD due to DN.

(ESRD) in many countries, with an ever increasing incidence and prevalence. ${ }^{1}{ }^{2}$ In general, its frequency as cause of ESRD has bypassed all other causes and ESRD due to $\mathrm{DN}$ is seen as one of the main reasons for the accelerating growth in patients with ESRD, thus contributing not only to an important loss of quality of life and early death but also to a vast increase in health expenditure worldwide. ${ }^{34}$

In the USA, for instance, the incidence of ESRD due to DN was 156 per million population (pmp) in 2011. ${ }^{4}$ Recently, a report from France found an incidence of $56 \mathrm{pmp}$ while this number was $27.6 \mathrm{pmp}$ in the $\mathrm{UK}^{5}{ }^{6}$ Although the incidence of renal replacement therapy (RRT) due to DN seemed to increase in most countries, in line with the increasing incidence of diabetes mellitus (DM) among the population, other countries reported a stabilisation or even a decrease..$^{5-9}$

The aim of the present study was to report any recent changes in the incidence and prevalence of RRT for DN in the Netherlands, taking into account the increased prevalence of $\mathrm{DM}$ in the general population from approximately 260000 in 1993, 466000 in 2000 to 815000 in 2011. ${ }^{10}$ Furthermore, we analysed the 
survival rates on RRT for ESRD due to DN between the periods 2000-2004 and 2005-2009.

\section{METHODS}

\section{Study design and aims}

This nationwide cohort study is the third 'Dutch diabetes estimates' (DUDE) study. The DUDE studies aim to investigate the magnitude and impact of DM and its complications in the Netherlands. Aim of the present study was to investigate the course of the incidence and prevalence of RRT for ESRD due to DN in the Netherlands in the period 2000-2012. Furthermore, survival among patients on RRT for ESRD due to DN in this period was analysed.

\section{Data collection}

Data from the RENINE registry were used. All Dutch centres $(n=69)$ providing RRT collaborated and shared their individual patient's data with the RENINE registry on a continuous basis, yielding a nationwide and $100 \%$ registration of treated patients. ${ }^{12}$

In the Dutch RENINE registry, information including primary renal disease necessitating RRT is registered according to the coding of the European Renal Association European Dialysis and Transplant Association (ERA-EDTA) registry. In this registry, DM as primary renal disease is registered under the EDTA codes 80 (Diabetes glomerulosclerosis or diabetic nephropathyType 1 diabetes mellitus) and 81 (Diabetes glomerulosclerosis or diabetic nephropathy-Type 2 diabetes mellitus). ${ }^{13}{ }^{14}$ In this manuscript, patients with either code 80 or code 81 as primary cause of ESRD necessitating RRT will be further referenced as patients with type 1 or type $2 \mathrm{DN}$ (T1DN or T2DN, respectively).

In the RENINE registry, RRT is defined as dialysis (peritoneal or haemodialysis) or kidney (also including kidney-pancreas $\mathrm{Tx}$ ) transplantation (Tx). Patients are only registered in the RENINE registry if they are using RRT for $>30$ days. The incidence of RRT was defined as the number of new patients per year and prevalence was defined as the number of patients alive and on RRT on 31 December of the relevant year. Both crude and the age and gender adjusted incidence, using the European Union (27 countries) age and gender distribution, were calculated. For both of these items, the mid-year population (30 June) was used as denominator. Owing to unknown or missing data regarding the cause of ESRD in some centres, additional sensitivity analyses were performed for the crude incidence in order to compare outcomes between centres with $<20 \%$ missing $(\mathrm{n}=38)$ and $\geq 20 \%$ ( $n=31)$ missing data.

\section{Statistical analysis}

Trends in time were analysed with Joinpoint regression. The Joinpoint regression technique allows identification of points in time where a significant change in the linear slope of a trend occurs. The analysis starts with zero joinpoints (ie, a straight line) and then tests whether one or more joinpoints are significantly different and must be added to the model.$^{15}$ The slope of a trend was calculated using the observed rate as the outcome variable and the year as the explanatory variable. The analysis was adjusted for changes in the age and gender distribution of the population. The annual percentage change (APC) was subsequently computed by the formula $\mathrm{APC}=[\exp (\beta)-1] \times 100$, where $\beta$ denotes the regression coefficient representing the estimated effect of time on the rate. A negative APC describes a decreasing trend and a positive APC describes an increasing trend. Joinpoint regression software (V.3.3) provided by the Surveillance Research Programme of the US National Cancer Institute was used to examine whether trends were linear.

Statistical analysis of unadjusted and adjusted survival, for patients starting RRT between 2000 and 2009, was performed by Cox proportional hazards regression. For the analysis of patient survival on dialysis the first day on dialysis was the starting point, the event studied was death and reasons for censoring were recovery of renal function, loss to follow-up, end-of-follow-up time and kidney transplantation. For the analysis of patient and graft survival after transplantation, the date of the first transplant was defined as the first day of follow-up. For the analysis of patient survival after transplantation, death was the event studied and for graft survival the events were graft failure and death. Reasons for censoring were loss-to-follow-up and end-of-follow-up period. SAS V.9.1 software was used for all statistical analyses.

\section{RESULTS}

\section{Crude incidence and prevalence}

In 2012, the total Dutch population consisted of 16730348 persons. ${ }^{16}$ Of all 2011 persons (120.2 pmp) starting RRT in the year 2012, 328 (19.6 pmp) needed RRT for ESRD due to DN. Over time, the crude incidence increased from $15.6 \mathrm{pmp}$ in 2000 to $19.6 \mathrm{pmp}$ in 2012 , increasing by $1.9 \%$ annually.

The crude prevalence of patients using RRT for all causes in the year 2012 was 15449 (923.4 pmp) of which 1817 (108.6 pmp) used RRT for ESRD due to DN. Over time, the crude prevalence increased at a slower rate: by $6.7 \%$ annually from 2000 to 2009 and by $2.6 \%$ annually from 2009 to 2012.

The crude incidence and prevalence of RRT for DN, both T1DN and T2DN, non-DN and unknown/missing causes of ESRD are presented in online supplementary appendix 1.

\section{Adjusted incidence}

The age and gender adjusted incidence of RRT for ESRD due to non-DN causes increased from 2000 to 2008 by $2 \%$ annually and remained stable after 2008 (table 1). As for RRT in ESRD due to DN, the incidence was stable over time, but trends were different by type of 


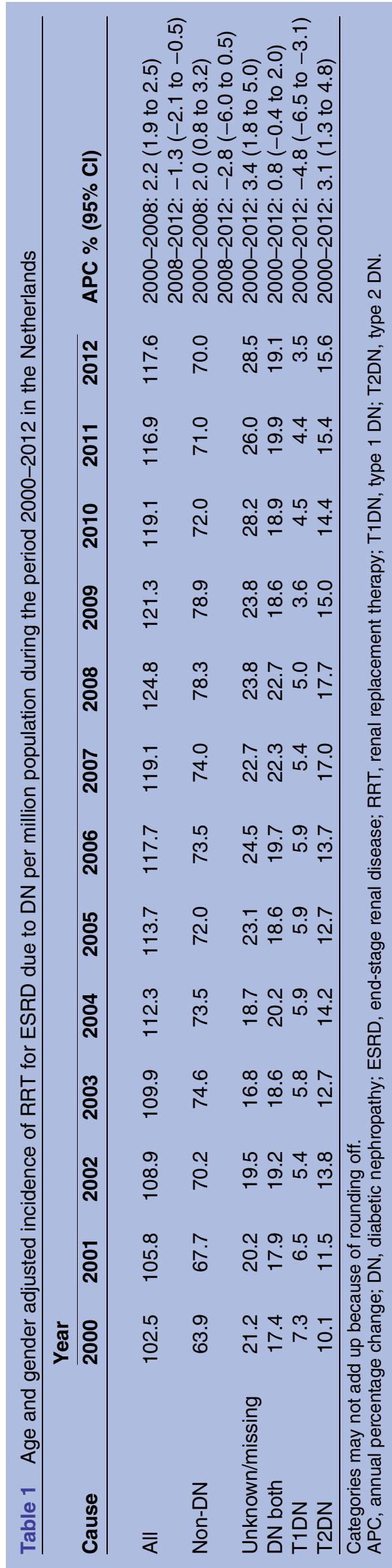

diabetes. There was an increase in incidence of RRT for T2DN from 2000 to 2012 by $3.1 \%$ annually, while the incidence of RRT for ESRD due to T1DN decreased in this period by $-4.8 \%$ annually.

Sensitivity analyses of the adjusted incidence in centres with $<20 \%$ and $\geq 20 \%$ missing or unknown primary causes of ESRD also showed an increasing incidence for T2DN and decreasing incidence for T1DN in both groups. Among centres with $<20 \%$ missing or unknown primary causes of ESRD, there was an annual increase of $2.1 \%$ in the incidence of RRT for ESRD due to both forms of DN in the period 2000 to 2012; however, it is stabilised in centres with $\geq 20 \%$ missing or unknown primary causes of ESRD (see online supplementary appendix 2A-D).

\section{Adjusted prevalence}

The age and gender adjusted prevalence of RRT for ESRD due to non-DN causes increased from 2000 to 2009 by $2.6 \%$ annually and remained stable after 2009 (table 2). Prevalence of RRT for DN increased from 2000 to 2009 by $5.8 \%$ annually and remained stable after 2009. For T2DN, prevalence increased at a declining rate: $12.4 \%$ in the period 2000 to 2003 and $8.7 \%$ in the period 2003 to 2009, and remained stable after 2009. For T1DN, prevalence increased from 2000 to 2010 by $1.2 \%$ and stabilised from 2010 onwards.

\section{Treatment modality}

From 2000 to 2012, adjusted incidence of dialysis for DN was stable due to an annual increase of $3.1 \%$ for T2DN and a decrease of $-6.1 \%$ for T1DN in this period (see online supplementary appendix 3A). Incidence of $\mathrm{Tx}$ for both forms of $\mathrm{DN}$ increased by $11.1 \%$ annually: $10.1 \%$ for T2 DN and $8.8 \%$ for T1DN. Prevalence of dialysis for DN increased from 2000 to 2008 by $5.9 \%$ annually and remained stable afterwards. For T2DN, prevalence of dialysis increased at a declining rate: from 2000 to 2003 by $12.5 \%$ annually to $7.2 \%$ in the period 2003 to 2009, and was stable after 2009. For T1DN, an initial decrease of prevalence in the period 2000 to 2010 by $-1.3 \%$ annually seemed to disappear after 2010 . Prevalence of patients with Tx increased from 2000 to 2004 by $4.6 \%$ annually and from 2004 to 2012 by $7.7 \%$. For T1DN there was an annual increase of $3.5 \%$ throughout the follow-up period, while for T2DN prevalence only increased between 2002 and 2010 by $18.7 \%$ annually (see online supplementary appendix 3B).

\section{Survival}

Survival and subsequent HRs were significantly worse for patients on RRT for ESRD due to DN compared to a non-DN reference population (table 3). Compared to patients with RRT for ESRD due to T2DN, patients with T1DN had a worse survival on dialysis (both for haemodialysis and peritoneal dialysis, see online supplementary appendix 4A,B) but not after kidney transplantation 


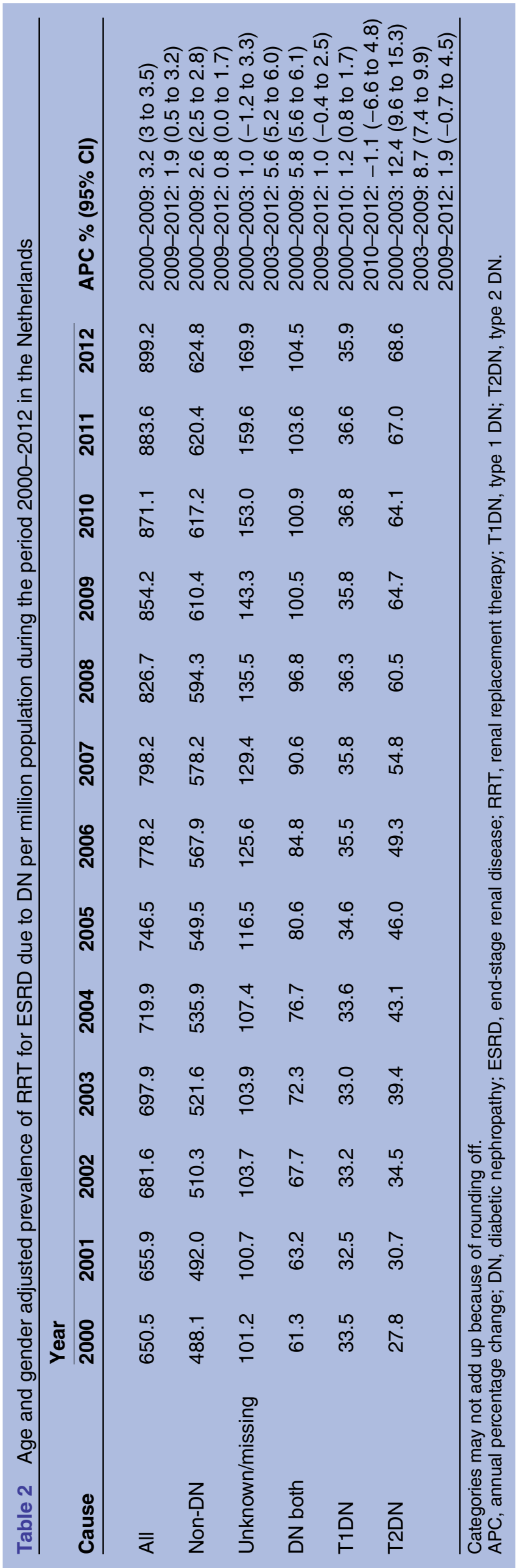

(also for graft survival, see online supplementary appendix 4C).

When comparing survival between patients who started RRT for ESRD due to DN in the period 2000-2004 and 2005-2009, patients in the latter time frame had significantly better outcomes (adjusted HR $0.76,95 \%$ CI 0.69 to 0.84 ). Their survival on dialysis was better (HR $0.77,95 \%$ CI 0.70 to 0.85 ) while there was a non-significant trend towards improvement in patient's survival Tx (HR 0.77 95\% CI 0.54 to 1.10). Both for T1DN and T2DN, survival on RRT improved in the period 2005-2009 as compared to 2000-2004: HR 0.75 (95\% CI 0.62 to 0.90 ) and HR 0.77 (95\% CI 0.69 to 0.87 ), respectively (see online supplementary appendix 4$)$.

\section{DISCUSSION}

We report a $4.8 \%$ average yearly decrease in age and gender adjusted annual incidence of RRT for ESRD due to T1DN and a $3.1 \%$ increase for T2DN in the period 2000 to 2012. The combined incidence of RRT for ESRD due to both types of DN has been stable over the period. Together with an increased survival over these years, this may indicate that the number of patients needing RRT for ESRD due to DN is stable and that these patients now live longer while on RRT.

These findings are remarkable, given the current increase in patients with T2DM in the Netherlands. Best estimations for T2DM show an increase from approximately 260000 in 1993, to 466000 in 2000 and to 815000 in 2011. ${ }^{10}{ }^{11}$ Although trends cannot be extrapolated one-on-one from observational data, we hypothesise it may suggest that the presently advocated approach with emphasis on early preventive measures (including lifestyle advices, stricter control of blood pressure and lipid profile disturbances, and improved glycaemic regulation), increased use of renoprotective medication (including ACE inhibitors or angiotensin II receptor antagonists), improved accessibility to RRT facilities or changes in healthcare delivery in the Netherlands may have had a positive impact. ${ }^{10}$ 17-20

Nevertheless, other explanations for our findings should be discussed. First and foremost, centres with unknown or missing data regarding the primary cause of ESRD may have confounded our findings. Indeed, sensitivity analyses showed different trends in the age and gender adjusted incidence of RRT for ESRD due to DN between centres with $<20 \%$ and $\geq 20 \%$ missing data, but the ones with fewer missing data confirmed our results. Second, a lag time in diagnosis of DN and development of ESRD should be taken into account. However, the transition rate from each stage of $\mathrm{DN}$ to the next one, of approximately $2.5 \%$ per year, should imply a higher prevalence of T2DN-related ESRD in the present study and this is quickly increasing disease prevalence in the general population. ${ }^{21}$ Third, the results could also indicate that, in line with other countries, in recent years more often a non-dialytic, conservative 
Table 3 Patient survival probabilities and crude HRs and age and gender adjusted HRs of patients starting RRT, subdivided into RRT (both dialysis and Tx), dialysis and kidney transplantation in the period 2000-2010

\begin{tabular}{|c|c|c|c|c|c|}
\hline & $\begin{array}{l}\text { Adjusted } 1 \text { year } \\
\text { survival }(95 \% \mathrm{Cl})\end{array}$ & $\begin{array}{l}\text { Adjusted } 5 \text { year } \\
\text { survival }(95 \% \mathrm{Cl})\end{array}$ & $\begin{array}{l}\text { Unadjusted } 5 \text { year } \\
\text { survival }(95 \% \mathrm{Cl})\end{array}$ & $\begin{array}{l}\text { Crude HR } \\
(95 \% \mathrm{Cl})\end{array}$ & $\begin{array}{l}\text { Adjusted } \\
\text { HR (95\% Cl) }\end{array}$ \\
\hline \multicolumn{6}{|c|}{ Survival on RRT } \\
\hline No DN & 90.2 (89.7 to 90.7$)$ & $62.1(61.1$ to 63.1$)$ & 53.6 (53.0 to 54.1$)$ & 1 (ref) & 1 (ref) \\
\hline DN both & $88.1(87.2$ to 89.1$)$ & 49.7 (47.9 to 51.6$)$ & 39.3 (38.6 to 40.1$)$ & $1.50(1.43$ to 1.58$)$ & $1.45(1.38$ to 1.53$)$ \\
\hline T1DN & 84.6 (82.2 to 87.0$)$ & 41.1 (37.7 to 44.8$)$ & 44.5 (43.0 to 45.9$)$ & 1.29 (1.18 to 1.40$)$ & 1.83 (1.68 to 2.00$)$ \\
\hline T2DN & 89.2 (88.1 to 90.3$)$ & 52.6 (50.5 to 54.8$)$ & 37.1 (36.3 to 37.9$)$ & 1.61 (1.52 to 1.70$)$ & 1.35 (1.27 to 1.43$)$ \\
\hline DN both & 86.5 (85.4 to 87.7$)$ & 43.3 (41.3 to 45.4$)$ & 35.0 (34.3 to 35.7$)$ & 1.26 (1.19 to 1.33$)$ & $1.32(1.25$ to 1.39$)$ \\
\hline T1DN & 83.2 (80.7 to 85.9$)$ & $33.8(30.2$ to 38.0$)$ & 35.0 (33.7 to 36.3$)$ & $1.28(1.17$ to 1.41$)$ & $1.72(1.57$ to 1.88$)$ \\
\hline T2DN & 87.6 (86.3 to 88.8$)$ & 46.4 (44.1 to 48.8$)$ & 35.0 (34.2 to 35.8$)$ & 1.25 (1.18 to 1.32$)$ & 1.21 (1.14 to 1.29$)$ \\
\hline \multicolumn{6}{|c|}{ Survival after kidney $T x$} \\
\hline No DN & 97.5 (97.1 to 98.0$)$ & 90.8 (90.0 to 91.8$)$ & 87.4 (86.5 to 88.3$)$ & 1 (ref) & 1 (ref) \\
\hline DN both & 94.4 (92.9 to 96.0$)$ & 83.2 (80.4 to 86.0$)$ & 74.6 (71.7 to 77.3$)$ & 2.07 (1.77 to 2.42 ) & 1.85 (1.58 to 2.17$)$ \\
\hline
\end{tabular}

treatment is chosen for patients with ESRD due to DN. ${ }^{22} 23$ Finally, the dramatic scenario of increasing mortality among patients with DM before initiating RRT could be hypothesised. However, as the life expectancy of Dutch patients with T2DM is reported to be stable, at a level equal to the general population, this latter scenario is not quite conceivable. ${ }^{20}$

In a previous Dutch report until 2002, the incidence rates of RRT were also increasing for T2DN and decreasing for T1DN. ${ }^{24}$ The current report extends the studied period and adds to these observations by demonstrating a stabilisation of ESRD due to DN. This is in accordance with reports from other registries: Sørensen et at reported a stabilisation in Danish patients with DM who were referred for RRT and recently a French study

Table 4 Incidence rates of ESRD due to DN: differences between countries

\begin{tabular}{lcc}
\hline Country & $\begin{array}{l}\text { Diabetes } \\
\text { national } \\
\text { prevalence (\%) }\end{array}$ & $\begin{array}{l}\text { Incidence rate of } \\
\text { ESRD due to DN } \\
\text { (pmp) }\end{array}$ \\
\hline Belgium & 6.63 & 37.8 \\
Denmark & 7.51 & 29.7 \\
France & 7.30 & 32.4 \\
Japan & 11.2 & 131.3 \\
The Netherlands & 7.31 & 18.6 \\
Norway & 5.85 & 14.5 \\
Spain & 8.14 & 29.3 \\
Sweden & 5.71 & 29.7 \\
USA & 10.94 & 159.2 \\
\hline Based on 2011 data derived from refs 4 and 26. \\
DN, diabetic nephropathy; ESRD, end-stage renal disease; pmp, \\
per million population.
\end{tabular}

showed that in the period 2007 to 2011, there was a decreasing incidence of RRT for ESRD due to T1DN and after an increase until 2009, a stabilisation for T2DN. ${ }^{6}$ A report from the USA also showed a decreasing incidence of ESRD treatment for DN. ${ }^{25}$

Nevertheless, notable differences in the rate and course of the incidence of RRT for ESRD due to DN between countries exist. In 2005, the mean annual increase of the age and gender adjusted incidence of RRT for T2DN in 10 European registries was estimated to be $12 \%$, with a variation of $7-21 \% .{ }^{13}$ Worldwide, the relative incidence rate of ESRD due to diabetes was reported as ranging from less than $20 \%$ in Russia and Norway to $60 \%$ in Singapore or Mexico. ${ }^{4}$ When comparing 2011 incidence data with other developed European countries with an equal $(\sim 7.5 \%)$ diabetes prevalence in the general population and Japan and the USA (table 4), the differences are still remarkable. ${ }^{4} 26$ Thus it seems that these differences may not be solely explained by variations in diabetes prevalence in the general population between countries. Finding explanations and subsequent solutions for these differences might help to alleviate the burden of ESRD due to DN.

Analysis of survival in the present study confirms previous findings that mortality is higher among patients with RRT for ESRD due to DN, in particular T1DN, compared to patients with RRT for ESRD due to a non-DN cause, implying an additional detrimental effect of diabetes induced end-organ damage. ${ }^{27} 28$ Regarding survival by modality, Schroijen et al reported almost the same crude HR for mortality (1.51) for patients with ESRD due to DN on dialysis in seven other European countries. $^{28}$ In accordance with previous reports, we found that renal transplantation yielded higher 
(approximately a doubling) survival rates than dialysis. ${ }^{4}$ Although individual patient data is lacking, this may well be due to the selection of healthier participants for renal transplantation as compared to dialysis. It should be noted that the survival rates among patients with DN using RRT have increased during the last decade. Although there could be different explanations for this finding, that is, better control of (cardiovascular) risk factors, improved dialysis techniques or a higher number of renal transplantations, this important finding adds to the aforementioned hypothesis that changes within the present healthcare system and delivery also contribute to improved care for patients with ESRD.

This study is subject to some limitations. As mentioned before, it cannot be ruled out that some of the patients with unknown/missing primary causes of RRT indeed had DN. It should also be taken into account that the classification of diabetes as primary cause of ESRD into T1DN and T2DN was made primarily by the attending nephrologist. Furthermore, the diagnosis of DN was made clinically and was rarely based on biopsy findings. Although this may have induced errors in classification, it reflects clinical practice. Finally, as no (longitudinal) individual patient data on health status was available we can only hypothesise about the influence of healthcare or patient-related characteristics on, for example, differences between patients with T1DN and T2DN. One possible hypothesis is that earlier identification of T1DN patients, with subsequent earlier surveillance for proteinuria and an earlier start of renoprotective medications, may explain observed differences between T1DN and T2DN. Furthermore, patients with T1DN, most likely being younger, are more often deemed suitable for Tx.

In conclusion, the incidence of RRT for ESRD due to DN was stable in the Netherlands over the last decade reflecting a decrease for T1DN and an increase for T2DN. The overall incidence and prevalence are also relatively low in comparison with most developed countries. Taken together with improved survival rates this may suggest that changes in healthcare have been successful with regard to the prevention and subsequent treatment of ESRD due to DN.

\author{
Author affiliations \\ ${ }^{1}$ Diabetes Centre, Isala, Zwolle, The Netherlands \\ ${ }^{2}$ Department of Medical Informatics, Academic Medical Center, University of \\ Amsterdam, ERA-EDTA Registry, Amsterdam, The Netherlands \\ ${ }^{3}$ Department of Internal Medicine, University of Groningen, University Medical \\ Centre Groningen, Groningen, The Netherlands \\ ${ }^{4}$ Department of Nephrology, Radboud University Medical Centre, Nijmegen, \\ The Netherlands \\ ${ }^{5}$ RENINE Registry, Leiden, The Netherlands \\ ${ }^{6}$ Department of Internal Medicine, Isala, Zwolle, The Netherlands
}

Contributors PRvD designed the study, researched data and wrote the first version of the manuscript and is responsible for the final version of the manuscript. AK researched data and contributed to writing the manuscript. SJJL, AJH, NK, KJJ, HJGB all contributed to the writing and critically revising the intellectual content of the manuscript. All authors contributed to the conception and design of the manuscript and interpretation of the data. All authors approved the final version of the manuscript for publication.
Funding This research received no specific grant from any funding agency in the public, commercial or not-for-profit sectors.

Competing interests None.

Provenance and peer review Not commissioned; externally peer reviewed.

Data sharing statement No additional data are available.

Open Access This is an Open Access article distributed in accordance with the Creative Commons Attribution Non Commercial (CC BY-NC 4.0) license, which permits others to distribute, remix, adapt, build upon this work noncommercially, and license their derivative works on different terms, provided the original work is properly cited and the use is non-commercial. See: http:// creativecommons.org/licenses/by-nc/4.0/

\section{REFERENCES}

1. Gall MA, Nielsen FS, Smidt UM, et al. The course of kidney function in type 2 (non-insulin-dependent) diabetic patients with diabetic nephropathy. Diabetologia 1993;36:1071-8.

2. Hallan $\mathrm{SI}$, Coresh J, Astor BC, et al. International comparison of the relationship of chronic kidney disease prevalence and ESRD risk. $J$ Am Soc Nephrol 2006;17:2275-84

3. Stel VS, van de Luijtgaarden MWM, Wanner C, et al. The 2008 ERAEDTA Registry Annual Report-a précis. NDT Plus 2011;4:1-13.

4. Collins AJ, Foley RN, Chavers B, et al. United States Renal Data System 2011 Annual Data Report: atlas of chronic kidney disease \& end-stage renal disease in the United States. Am J Kidney Dis 2012;59:A7, e1-420.

5. Hill CJ, Fogarty DG. Changing trends in end-stage renal disease due to diabetes in the United Kingdom. $J$ Ren Care 2012;38(Suppl 1):12-22.

6. Assogba FGA, Couchoud C, Hannedouche T, et al. Trends in the epidemiology and care of diabetes mellitus-related end-stage renal disease in France, 2007-2011. Diabetologia 2014;57:718-28.

7. Grace BS, Clayton $P$, McDonald SP. Increases in renal replacement therapy in Australia and New Zealand: understanding trends in diabetic nephropathy. Nephrol Carlton Vic 2012;17:76-84.

8. De Boer IH, Rue TC, Hall YN, et al. Temporal trends in the prevalence of diabetic kidney disease in the United States. JAMA 2011;305:2532-9.

9. Sørensen VR, Hansen PM, Heaf J, et al. Stabilized incidence of diabetic patients referred for renal replacement therapy in Denmark. Kidney Int 2006;70:187-91.

10. Houweling ST, Kleefstra N, Meulepas M, et al. New estimates of diabetes prevalence in the Netherlands, based on information from 5 million subjects (DUDE-1). Diabetologia 2013;56:291.

11. Baan $C A$, Bonneux $L$, Ruwaard $D$, et al. The prevalence of diabetes mellitus in the Netherlands: a quantitative review. Eur J Public Health 1998;8:210-16.

12. de Charro FT, Ramsteyn PG. RENINE, a relational registry. Registratie Nierfunctievervanging Nederland. Nephrol Dial Transplant 1995;10:436-41.

13. Van Dijk PCW, Jager KJ, Stengel B, et al. Renal replacement therapy for diabetic end-stage renal disease: data from 10 registries in Europe (1991-2000). Kidney Int 2005;67:1489-99.

14. Van Dijk PC, Jager KJ, de Charro F, et al. Renal replacement therapy in Europe: the results of a collaborative effort by the ERA-EDTA registry and six national or regional registries. Nephrol Dial Transplant 2001;16:1120-9.

15. Kim HJ, Fay MP, Feuer EJ, et al. Permutation tests for Joinpoint regression with applications to cancer rates. Stat Med 2000;19:335-51.

16. http://statline.cbs.nl/StatWeb/publication/?VW=T\&DM=SLNL\& $P A=37713 \& H D=140429-1042$ (accessed 1 May 2014).

17. van Hateren KJJ, Drion I, Kleefstra N, et al. A prospective observational study of quality of diabetes care in a shared care setting: trends and age differences (ZODIAC-19). BMJ Open 2012;2: pii: e001387.

18. Parving $\mathrm{H}-\mathrm{H}$, Hovind $\mathrm{P}$. Microalbuminuria in type 1 and type 2 diabetes mellitus: evidence with angiotensin converting enzyme inhibitors and angiotensin II receptor blockers for treating early and preventing clinical nephropathy. Curr Hypertens Rep 2002;4:387-93.

19. Ubink-Veltmaat LJ, Bilo HJG, Groenier $\mathrm{KH}$, et al. Prevalence, incidence and mortality of type 2 diabetes mellitus revisited: a prospective population-based study in The Netherlands (ZODIAC-1). Eur J Epidemiol 2003;18:793-800.

20. Lutgers HL, Gerrits EG, Sluiter WJ, et al. Life expectancy in a large cohort of type 2 diabetes patients treated in primary care (ZODIAC-10). PLOS ONE 2009;4:e6817. 
21. Adler Al, Stevens RJ, Manley SE, et al. Development and progression of nephropathy in type 2 diabetes: the United Kingdom Prospective Diabetes Study (UKPDS 64). Kidney Int 2003:63:225-32.

22. Jassal SV, Kelman EE, Watson D. Non-dialysis care: an important component of care for elderly individuals with advanced stages of chronic kidney disease. Nephron Clin Pract 2011;119(Suppl 1):c5-9.

23. Crail S, Walker R, Brown M, et al. Renal supportive and palliative care: position statement. Nephrol Carlton Vic 2013;18:393-400.

24. Gansevoort RT, van der Heij B, Stegeman CA, et al. Trends in the incidence of treated end-stage renal failure in the Netherlands: hope for the future? Kidney Int Supp/ 2004:S7-10.
25. Burrows NR, Li Y, Geiss LS. Incidence of treatment for end-stage renal disease among individuals with diabetes in the U.S. continues to decline. Diabetes Care 2010;33:73-7.

26. International Diabetes Federation. IDF diabetes atlas. 5th edn. Brussels, Belgium: International Diabetes Federation, 2011.

27. Lok CE, Oliver MJ, Rothwell DM, et al. The growing volume of diabetes-related dialysis: a population based study. Nephrol Dial Transplant2004;19:3098-103.

28. Schroijen MA, van de Luijtgaarden MWM, Noordzij M, et al. Survival in dialysis patients is different between patients with diabetes as primary renal disease and patients with diabetes as a co-morbid condition. Diabetologia 2013;56:1949-57. 